\title{
Solution of the Black-Scholes Equation: LDM and SDM
}

\author{
R. K. Pavan Kumar. Pannala
}

Department of Mathematics, Chandigarh University, Gharuan-Mohali, Punjab-140413, India.

Email: kamesh9.iit@gmail.com

\begin{abstract}
The main aim of this paper is to discuss a new way of a non-discretization method for the solution of the BlackScholes equation. Black-Scholes is a mathematical model based on a partial differential equation. The solution of the model is of utmost importance in financial mathematics to estimate option pricing. Several analytical, numerical, and non-discretization methods are existing in the literature to solve the model. Two decomposition methods namely the Laplace decomposition method (LDM) and Sumudu decomposition method (SDM) are adopted for the present study. The results of the present techniques have closed agreement with an approximate solution which has been obtained with the help of the Adomian Decomposition Method (ADM).
\end{abstract}

Keywords: Black-Scholes equation, Decomposition method, Laplace transform, Stock prices, Sumudu transform

\section{Introduction}

Financing, in the modern-day, has become more complex and involves precision concerning the pricing of instruments that are used to fund businesses. This is more so considering the ever-evolving modes of funding business projects. Dependence on a mathematical model in arriving at the issue price has become imperative. This evolution has made mathematical modeling and their testing on computer-generated simulations mandatory for business managers.

Myron Scholes, Robert Merton, and Fisher black had a significant breakthrough in the early 1970s about the pricing of complex financial instruments by introducing what is known as the Black-Scholes model. The significance of their model captured the world's attention when Myron Scholes and Robert Merton won the Nobel Prize for Economics in 1997. It was unfortunate that Fisher Black was not alive to join the celebrations of the noble prize (Hull, 2000). The Black-Scholes model has shown the world that it plays a significant role in the field of finance and paved the way for the growth of financial mathematics or financial engineering.

The partial differential equation of Black-Scholes is written as (Bohner \& Zheng, 2009; Fischer Black and Myron Scholes, 1973)

$u_{t}+a x^{2} u_{x x}+b x u_{x}-r u=0$

where $a=\frac{\sigma^{2}}{2}$ and $b=r-\delta, \mathrm{r}$ is the risk-free rate, $\sigma$ is the volatility and $\delta$ is the dividend yield.

In this work, the Laplace decomposition method (LDM) and Sumudu decomposition method (SDM) have used to study the Black-Sholes equations for a European option pricing problem consisting of (1.1) and the initial condition

$$
u(x, 0)=g(x)
$$

Assume that $\mathrm{g}(\mathrm{x})$ has derivatives of all orders. The LDM and SDM were introduced by Khuri (Khuri, 2001) and Eltayeb, et. al. (Eltayeb \& Kiliçman, 2012) respectively. And in these methods, the solution is considered as the summation of an infinite series that usually converges rapidly to the exact solutions.

\section{Description of the Laplace and Sumudu decomposition methods}

\subsection{Description of LDM}

The main aim of this section is to discuss the use of the Laplace transform algorithm for the nonlinear partial differential equations.

Consider the general form of nonlinear partial differential equation (1.1) with initial condition is given below

$$
L u+R u+N u=0
$$

with initial condition $u(x, 0)=g(x)$

where $u(x, t)$ is the unknown function, $\mathrm{L}$ is the linear differential operator $L=\frac{\partial}{\partial t}$, $\mathrm{R}$ is the remaining linear differential operator of the order less than $\mathrm{L}, \mathrm{Nu}$ represents the nonlinear terms. 
The methodology consists of applying Laplace transform $\mathcal{L}[u(x, t)]=u(x, s)$ on both sides of equation (2.1)

$$
\mathcal{L}[L u(x, t)]+\mathcal{L}[R u(x, t)]+\mathcal{L}[N u(x, t)]=\mathcal{L}[0]
$$

Using the differentiation property of Laplace transform

$$
\begin{aligned}
& s \mathcal{L}[u(x, t)]-g(x)+\mathcal{L}[R u(x, t)]+\mathcal{L}[N u(x, t)]=0 \\
& \mathcal{L}[u(x, t)]=\frac{g(x)}{s}+\frac{1}{s}\{-\mathcal{L}[R u(x, t)]-\mathcal{L}[N u(x, t)]\}
\end{aligned}
$$

The second step in the Laplace decomposition method is that, represent the solution as an infinite series given below

$$
u=\sum_{n=0}^{\infty} u_{n}(x, t)
$$

The nonlinear operator is decomposed as

$$
N u=G(u)=\sum_{n=0}^{\infty} A_{n}
$$

where $A_{n}$ are Adomian polynomials (Biazar \& Shafiof, 2007) of $u_{0}, u_{1}, u_{2}, \ldots . u_{n}$ and it can be determined formally as follows

$$
A_{n}=\frac{1}{n !}\left[\frac{d^{n}}{d \lambda^{n}}\left\{N\left(\sum_{i=0}^{\infty} \lambda^{i} u_{i}\right)\right\}\right]_{\lambda=0}
$$

The first three Adomian polynomials using (Biazar \& Shafiof, 2007) are:

$$
\begin{aligned}
A_{0} & =G\left(u_{0}\right) \\
A_{1} & =\left[\frac{d}{d \lambda} G\left(u_{0}+u_{1} \lambda\right)\right]_{\lambda=0}=u_{1} G^{\prime}\left(u_{0}\right) \\
A_{2} & =\frac{1}{2}\left[\frac{d}{d \lambda}\left\{\left(u_{1}+2 u_{2} \lambda\right) G^{\prime}\left(u_{0}+u_{1} \lambda\right)\right\}\right]_{\lambda=0}=u_{2} G^{\prime}\left(u_{0}\right)+\frac{u_{1}^{2}}{2 !} G^{\prime \prime}\left(u_{0}\right) \\
A_{3} & =\frac{1}{3}\left[\frac{d}{d \lambda}\left\{\left(u_{2}+3 u_{3} \lambda\right) G^{\prime}\left(u_{0}+u_{1} \lambda\right)+\frac{\left(u_{1}+2 u_{2} \lambda\right)^{2}}{2 !} G^{\prime \prime}\left(u_{0}+u_{1} \lambda\right)\right\}\right]_{\lambda=0} \\
& =u_{3} G^{\prime}\left(u_{0}\right)+u_{1} u_{2} G^{\prime \prime}\left(u_{0}\right)+\frac{u_{1}^{3}}{3 !} G^{\prime \prime \prime}\left(u_{0}\right)
\end{aligned}
$$

From equations (2.5), (2.6), and (2.7) the equation (2.4) can be derived as

$$
\begin{aligned}
& \mathcal{L}\left[\sum_{n=0}^{\infty} u_{n}(x, t)\right]=\frac{g(x)}{s}+\frac{1}{s}\left\{-\mathcal{L}[R u(x, t)]-\mathcal{L}\left[\sum_{n=0}^{\infty} A_{n}\right]\right\} \\
& \sum_{n=0}^{\infty} \mathcal{L}\left[u_{n}(x, t)\right]=\frac{g(x)}{s}+\frac{1}{s}\left\{-\mathcal{L}\left[R u_{n}(x, t)\right]-\mathcal{L}\left[\sum_{n=0}^{\infty} A_{n}\right]\right\}
\end{aligned}
$$

On comparing both sides of the equation (2.10)

$$
\begin{aligned}
& \mathcal{L}\left[u_{0}(x, t)\right]=\frac{g(x)}{s}=K(x, s) \\
& \mathcal{L}\left[u_{1}(x, t)\right]=\frac{-1}{s} \mathcal{L}\left[R u_{0}(x, t)\right]-\frac{1}{s^{2}} \mathcal{L}\left[A_{0}\right] \\
& \mathcal{L}\left[u_{n+1}(x, t)\right]=\frac{-1}{s} \mathcal{L}\left[R u_{n}(x, t)\right]-\frac{1}{s^{2}} \mathcal{L}\left[A_{n}\right], n \geq 0
\end{aligned}
$$

$u_{0}, u_{1}, \ldots . u_{n}, \ldots$. are obtained by applying inverse Laplace transform to equations (2.11)-(2.13).

\subsection{Description of SDM}

By applying the Sumudu transform adopted from (Watugala, 1993) and (Belgacem et al., 2003) on (2.1)

$$
S[u(x, t)]=g(x)-v S\{[R u(x, t)]+[N u(x, t)]\}
$$

Using Adomian polynomials equations (2.14) can be written as

$$
\sum_{n=0}^{\infty} S\left[u_{n}(x, t)\right]=g(x)+v\left\{-S\left[R u_{n}(x, t)\right]-S\left[\sum_{n=0}^{\infty} A_{n}\right]\right\}
$$

$u_{0}, u_{1}, u_{2}, \ldots, u_{n}, \ldots$. are obtained by applying inverse Sumudu transform on (2.15) 


\section{Numerical example}

\subsection{Solution using LDM}

Consider the equation (1.1) with $a=1, b=\frac{1}{2}, r=1$ and $g(x)=x^{3}$ (Allahviranloo, 2013) then the equation becomes

$$
\begin{aligned}
& u_{t}+x^{2} u_{x x}+\frac{x}{2} u_{x}-u=0 \\
& \text { with } u(x, 0)=x^{3}
\end{aligned}
$$

The exact solution is $u(x, t)=x^{3} e^{\frac{-13 t}{2}}$

By applying the Laplace transform to (3.1)

$$
u(x, s)=\frac{x^{3}}{s}+\frac{1}{s} \mathcal{L}\left[u-x^{2} u_{x x}-\frac{x}{2} u_{x}\right]
$$

By applying inverse Laplace transform to (3.4)

$$
u(x, t)=\mathcal{L}^{-1}\left[\frac{x^{3}}{s}\right]+\mathcal{L}^{-1}\left[\frac{1}{s} \mathcal{L}\left\{u-x^{2} u_{x x}-\frac{x}{2} u_{x}\right\}\right]
$$

Let the solution of (3.5) is

$$
\sum_{n=0}^{\infty} u_{n}=x^{3}+\mathcal{L}^{-1}\left[\frac{1}{s} \mathcal{L}\left\{\sum_{n=0}^{\infty} A_{n}(u)\right\}\right]
$$

where $A_{n}(u)$ are Adomian polynomials

$$
\begin{aligned}
& \text { Let } u_{0}=x^{3} \\
& A_{0}=u_{0}-x^{2} \frac{\partial^{2} u_{0}}{\partial x^{2}}-\frac{x}{2} \frac{\partial u_{0}}{\partial x}=\frac{-13}{2} x^{3} \\
& u_{n+1}=\mathcal{L}^{-1}\left[\frac{1}{s} \mathcal{L}\left\{\sum_{n=0}^{\infty} A_{n}(u)\right\}\right] \\
& u_{1}=\frac{-13}{2} x^{3} t, u_{2}=\frac{\left(\frac{13}{2}\right)^{2} x^{3} t^{2}}{2 !}, u_{3}=-\frac{\left(\frac{13}{2}\right)^{3} x^{3} t^{3}}{3 !} \ldots \ldots \ldots
\end{aligned}
$$

Hence the solution of (3.1) up to ( $\mathrm{n}+1)$-terms is

$$
\begin{aligned}
u(x, t) & =u_{0}+u_{1}+u_{2}+u_{3}+\cdots+u_{n} \\
& =x^{3}\left[1-\left(\frac{13}{2}\right) t+\frac{\left(\frac{13}{2}\right)^{2} t^{2}}{2 !}-\frac{\left(\frac{13}{2}\right)^{3} t^{3}}{3 !}+\cdots+\frac{(-1)^{n}\left(\frac{13}{2}\right)^{n} t^{n}}{n !}\right]
\end{aligned}
$$

\subsection{Solution using SDM}

Using the equations (2.14)-(2.15), equation (3.1) along with (3.2) can be written as

$$
\begin{aligned}
& \sum_{n=0}^{\infty} u_{n}=S^{-1}\left\{x^{3}\right\}+S^{-1}\left[v S\left\{\sum_{n=0}^{\infty} A_{n}(u)\right\}\right]=x^{3}+S^{-1}\left[v S\left\{\sum_{n=0}^{\infty} A_{n}(u)\right\}\right] \\
& u_{0}=x^{3} \\
& u_{n+1}=S^{-1}\left[v S\left\{\sum_{n=0}^{\infty} A_{n}(u)\right\}\right] \\
& u_{1}=S^{-1}\left[v S\left(A_{0}\right)\right]=S^{-1}\left[v S\left(\frac{-13}{2} x^{3}\right)\right]=\left(\frac{-13}{2} x^{3}\right) S^{-1}[v S(1)]=\left(\frac{-13}{2} x^{3}\right) t \\
& u_{2}=S^{-1}\left[v S\left(A_{1}\right)\right]=S^{-1}\left[v S\left\{\left(\frac{13}{2}\right)^{2} x^{3} t\right\}\right]=\left(\frac{13}{2}\right)^{2} x^{3} \frac{t^{2}}{2 !}
\end{aligned}
$$

The solution of the equation (3.1) using the Sumudu Decomposition method up to $(\mathrm{n}+1)$ terms is $u(x, t)=u_{0}+u_{1}+u_{2}+u_{3}+\cdots+u_{n}$

$$
=x^{3}\left[1-\left(\frac{13}{2}\right) t+\frac{\left(\frac{13}{2}\right)^{2} t^{2}}{2 !}-\frac{\left(\frac{13}{2}\right)^{3} t^{3}}{3 !}+\cdots+\frac{(-1)^{n}\left(\frac{13}{2}\right)^{n} t^{n}}{n !}\right]
$$

The solutions obtained in (3.11) and (3.14) are the same

The below graph represents the analytical solution of the Black-Scholes partial differential equation (3.1) for $x=0.1: 0.1: 1$ and $t=0.1: 0.1: 1$ 


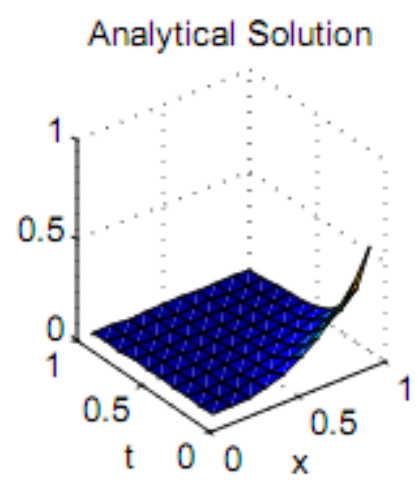

The following graphs represent the approximate solution of the Black-Scholes partial differential equation (3.1), which has been solved by ADM and Transform Decomposition Methods (LDM and SDM) for $x=$ 0.1: $0.1: 1$ and $t=0.1: 0.1: 1$, for various values of $n$.

$n$

4

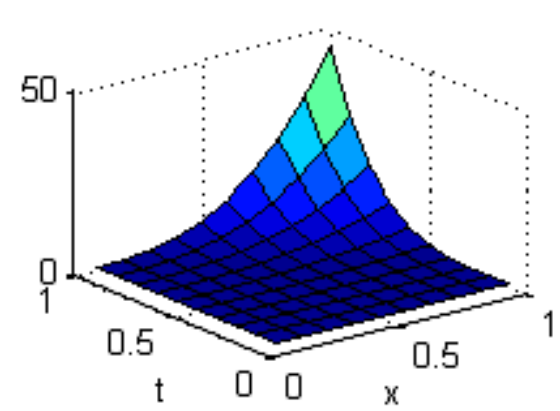

10

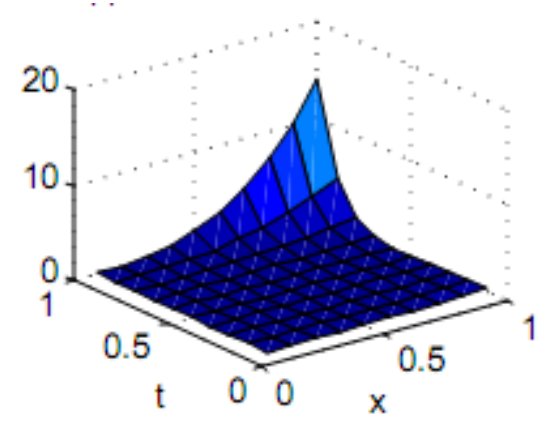

20

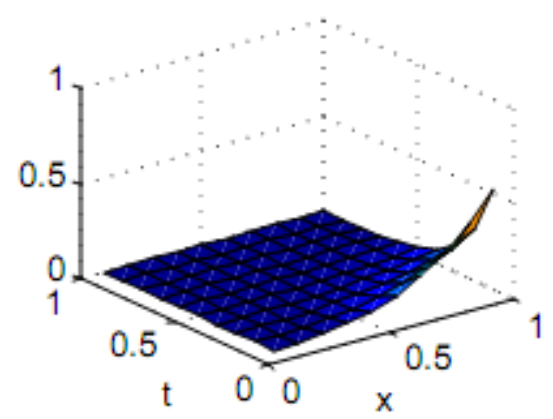

Approximate solution by LDM \& SDM
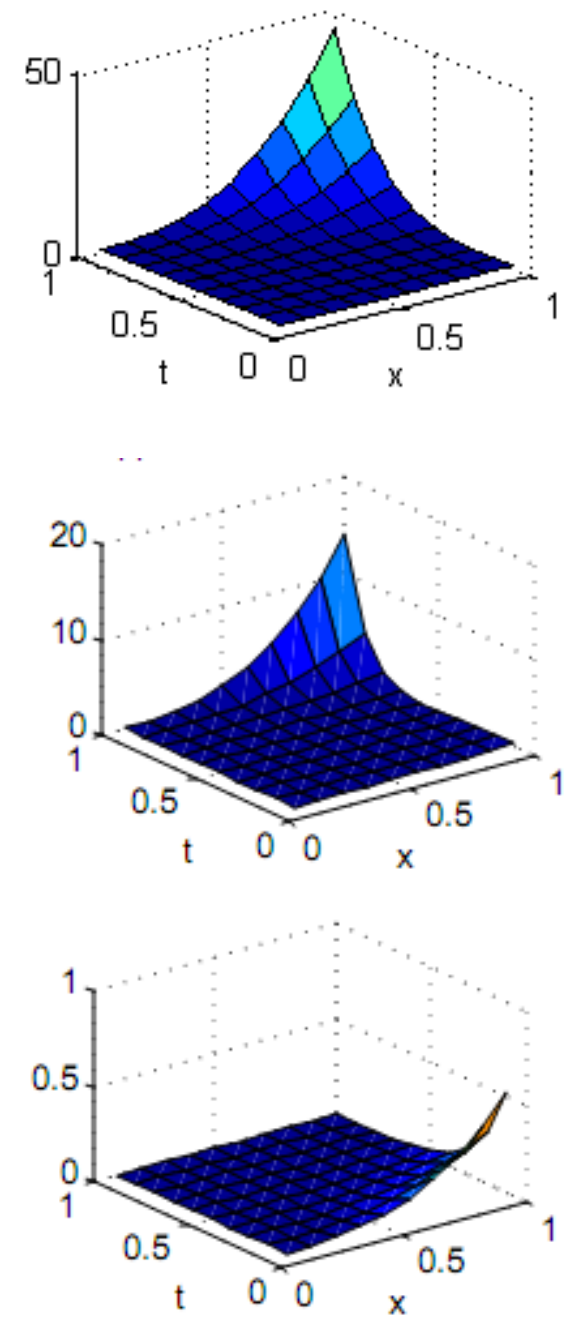

\section{Conclusion}

From the results, it has been found that the two methods Laplace decomposition (LDM) and Sumudu decomposition (SDM) methods can be used alternatively for the solution of the Black-Scholes equation that has a closed agreement with the Adomian decomposition (ADM) method (Ali, 2012; Allahviranloo, 2013) which is very much evident from the graphs. 


\section{References}

Ali, E. J. (2012). A new technique of initial boundary value problems using adomian Decomposition Method. International Mathematical Forum, 7(17), 799-814.

Allahviranloo, T. (2013). The use of iterative methods for solving Black-Scholes equation. 5(1), 1-11.

Belgacem, F. B. M., Karaballi, A. A., \& Kalla, S. L. (2003). Analytical investigations of the Sumudu transform and applications to integral production equations. Mathematical Problems in Engineering, 2003(3), 103118 .

Biazar, J., \& Shafiof, S. M. (2007). A simple algorithm for calculating Adomian polynomials. International Journal of Contemporary Mathematical Sciences, 2(20), 975-982. https://doi.org/10.12988/ijcms.2007.07099

Bohner, M., \& Zheng, Y. (2009). On analytical solutions of the Black-Scholes equation. Applied Mathematics Letters, 22(3), 309-313. https://doi.org/10.1016/j.aml.2008.04.002

Eltayeb, H., \& Kiliçman, A. (2012). Application of sumudu decomposition method to solve nonlinear system of partial differential equations. Abstract and Applied Analysis, 2012. https://doi.org/https://doi.org/10.1155/2012/412948

Fischer Black and Myron Scholes. (1973). The Pricing of Options and Corporate Liabilities. Journal of Political Economy, 81(3), 637-654. https://www.jstor.org/stable/1831029

Hull, J. C. (2000). Options, futures and other derivatives (5th ed.). Prentice Hall, New Jersey.

Khuri, S. A. (2001). A Laplace decomposition algorithm applied to a class of nonlinear differential equations. Journal of Applied Mathematics, 1(4), 141-155. https://doi.org/10.1155/S1110757X01000183

Watugala, G. K. (1993). Sumudu transform: A new integral transform to solve differential equations and control engineering problems. International Journal of Mathematical Education in Science and Technology, 24(1), 35-43. https://doi.org/10.1080/0020739930240105 